\title{
Diseño y construcción de un sistema automatizado de control de bombas de agua en un cultivo hidropónico en el entorno Arduino, UNSCH - Ayacucho
}

\author{
Jose Luis Huayanay villar ${ }^{1,2}$, Julio Ore Garcia ${ }^{2}$, Sheyla Yassira Meneses Hiyo ${ }^{2}$ \\ ${ }^{1}$ Universidad do Estado de Río de Janeiro, São Francisco Xavier, Río de Janeiro, Brasil \\ 2 Universidad Nacional de San Cristóbal de Huamanga, Portal Independencia 57, Ayacucho, Perú
}

Recibido el 8 de junio del 2020. Revisado el 2 de octubre del 2020. Aceptado el 5 de octubre del 2020.

DOI: https://doi.org/10.33017/RevECIPeru2020.0010/

\section{Resumen}

El objetivo del trabajo es desarrollar un sistema automatizado de riego en torno al Arduino para cultivos hidropónicos. El sistema utiliza una placa Arduino ATmega328P a la que se conecta diferentes componentes, tales como un módulo de reloj en tiempo real, pantalla LCD I2c, dos módulos relé de un canal y resistencias eléctricas; con la finalidad de obtener un sistema de micro controlador programable que puede activar y desactivar la bomba de agua de un invernadero en tiempos determinados de acuerdo al desarrollo del cultivo, particularmente en los cultivos de lechugas con diferentes variedades, para ello es necesario utilizar diferentes técnicas de control y realizar varias pruebas experimentales con el fin de ajustar ciertos parámetros de control, especialmente la humedad y temperatura. Este sistema fue instalado en el invernadero de la Comunidad de Mollepata en Ayacucho, dentro del convenio tria-anual "Inti, la energía que alimenta la Tierra" entre la Universidad Nacional de San Cristóbal de Huamanga (UNSCH) y el Comité Regional de Educación para el Desarrollo Internacional de Lanaudière (CREDIL-JOLIETTE) - Canadá.

Descriptores: Arduino Mega 328, módulo reloj en tiempo real, cultivos hidropónicos, sistema automatizado.

\section{Abstract}

The objective of the work to develop an automated irrigation system about Arduino for hydroponic crops The system uses an Arduino ATmega328P board to connect different components, such as a real-time clock module, LCD I2c screen, two one-channel relay modules and electrical resistors; For the purpose to obtain a micro programmable controller that turns on and off the water pump of a greenhouse in times determined according to the development of the crop, particularly in lettuce crops with different varieties .For this, it's necessary to use different control techniques and to carry out several experimental tests in order to adjust certain control parameters, especially humidity and temperature. This system was installed in the greenhouse of the Community of Mollepata in Ayacucho, within the three-year agreement "Inti, the energy that feeds the Earth" between the National University of San Cristóbal de Huamanga (UNSCH) and the Regional Committee of Education for Development Lanaudière International (CREDIL - JOLIETTE) - Canada.

Keywords: Arduino Mega 328, real-time clock module, hydroponic crops, automated system.

\section{Introducción}

Actualmente vemos que la sociedad está influenciada por factores sociales que impulsan el uso de la tecnología que está en continua evolución, ello hace posible replantear los modelos ya existentes para poder mejorarlos y proponer otros para nuestro bienestar.

La compañía Arduino es una comunidad tecnológica que diseña y manufactura placas de 
desarrollo de hardware, compuestas por micro controladores, elementos pasivos y activos. En el Perú hay antecedentes de que instituciones como Naylamp Mecatronics S.A.C y Electrotec Perú, vienen desarrollando proyectos multidisciplinarios en "el entorno Arduino [3][4][5]". Este micro controlado puede detectar el entorno mediante la recepción de entradas desde una variedad de sensores [4], asimismo podemos usar esta tecnología para el desarrollo de un sistema automatizado de control de bomba de agua en un cultivo hidropónico de diferentes variedades de lechugas, utilizando el agua como elemento esencial para aprovechar el potencial de la tierra[2]; su empleo y gestión constituyen un factor fundamental para elevar la productividad de la agricultura y asegurar una producción previsible.

La inversión en ciencia y tecnología agrícola, expresada en términos de investigación y servicios de extensión, ha demostrado tener una gran relevancia en el aumento del rendimiento de los cultivos agrícolas y la reducción de la pobreza en los países en desarrollo. Sin embargo, es importante que esta inversión esté definida según las necesidades de conocimiento expresadas por todos los actores involucrados [5].

El cultivo hidropónico se basa fundamentalmente en el desarrollo de las hortalizas en 8 canales de PVC dispuestos en cada parcela, llamados canales de cultivo, donde pasa un bajo caudal de agua con una pequeña cantidad de solución nutritiva la cual fluye hacia las raíces de las lechugas manteniéndolas nutridas, hidratadas, oxigenadas y de esta manera en constante desarrollo. Cada canal de cultivo tiene agujeros, donde se colocan las plantas, estos canales están apoyados sobre caballetes de fierro y madera que tiene una ligera pendiente o desnivel que facilita la circulación de la solución nutritiva. La solución es recolectada y almacenada en un tanque de polietileno y mediante la bomba de agua que lleva la solución hasta los canales de cultivo [1].

En general estos sistemas requieren de un menor número de horas de trabajo que los sistemas convencionales de producción, ya que no solo pueden automatizarse, sino que además la naturaleza de las tareas es sensiblemente diferente en estos sistemas. Además, en general las tareas son más livianas que en los sistemas convencionales, por lo que puede existir un ahorro sensible en mano de obra y por lo tanto en costos [1] [2].
Las mayores posibilidades tecnológicas del control de las condiciones de operación, su accesibilidad tecnológica y económica, baja contaminación, mejor aprovechamiento energético, bajo costo y otros factores, juegan a favor de estos sistemas de cultivo. Esperemos que en esta área, nuestro país alcance un rápido desarrollo agrícola.

El trabajo se desarrolló como un aporte al convenio tri-anual "Inti, la energía que alimenta la Tierra" entre la Universidad Nacional de San Cristóbal de Huamanga (UNSCH) y el Comité Regional de Educación para el Desarrollo Internacional de Lanaudière (CREDIL-JOLIETTE) - Canadá. Y fue puesta en funcionamiento en la empresa "Hidroponía -Ayacucho S.A."

\section{Metodología}

Para el diseño y construcción del sistema automatizado se ha utilizado la placa Arduino, un microcontrolador de código abierto basada en el microcontrolador microchip ATmega328P y desarrollada por Arduino.cc [5]. El Arduino posee 14 pinos digitales, 6 pinos analógicas, un resonador cerámico de $16 \mathrm{MHz}$ y conexión USB.

La tarjeta contiene todo lo necesario para el funcionamiento del micro controlador ATmega328P que está alimentada con voltaje $12 \mathrm{~V}$ y $1,3 \mathrm{~A}$ con corriente máxima entrada/salida $40 \mathrm{~mA}$ [4].

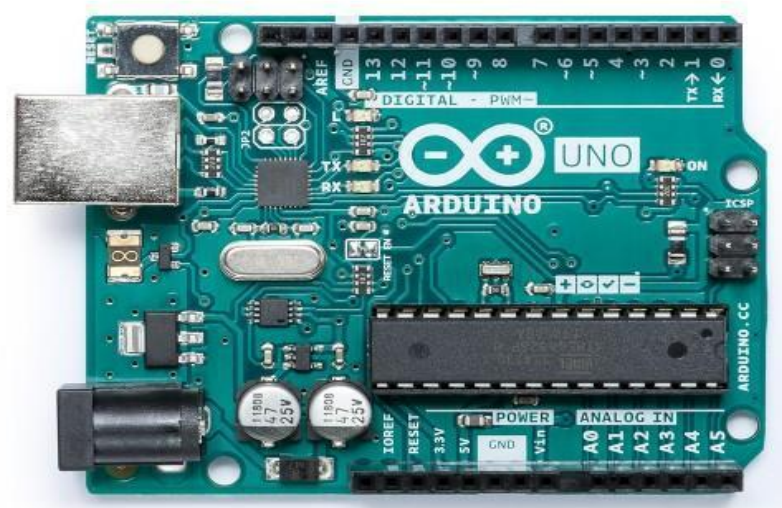

Figura 1: Pinos y partes del detalle del Arduino [4]

Asimismo, se ha utilizado "el entorno de desarrollo integrado (IDE) de Arduino", el cual es una aplicación multiplataforma (para Windows, macOS, Linux) que está escrita en el lenguaje de programación Java. Lo cual se ha utilizado para escribir y cargar la programación en la placa Arduino [3]5].

\subsection{Modulo reloj}


Para el proyecto fue necesario incluir el módulo reloj que está basado en el RTC DS3231 de Maxi y la EEPROM AT24C32 de ATMEL. Ambos circuitos integrados comparten el mismo bus de comunicación con el protocolo, este protocolo es de la pantalla Icd a utilizarse (Tabla 1). EI RTC DS3231 es la evolución del clásico RTC DS1307.

Este módulo utiliza un oscilador interno compensado por temperatura, lo que hace que su precisión sea muy alta. La memoria EEPROM AT24C32 permite almacenar 32Kbits de datos de manera permanente [4].

Este módulo hará posible el control de los tiempos programados de encendido y apagado del sistema (8 minutos cada 30-60 minutos aproximadamente). Este proceso se cumple de manera cíclica de día y de noche.

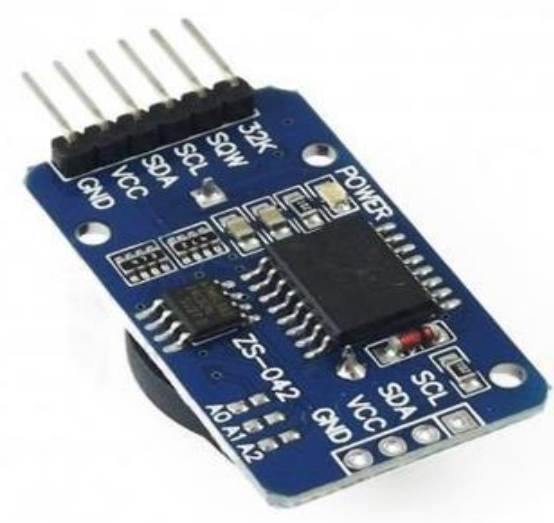

Figura 2: Imagen del módulo reloj (tienda Naylamp Mecatronics Perú)

Conexión y funcionamiento

- Voltaje de Operación: 3,3 V - 5 V

- Exactitud del reloj: 2 ppm

- Dirección I2C del DS3132: lectura (11010001), escribir (11010000)

- Memoria EEPROM AT24C32 (32Kbit)

- Salida de onda cuadrada programable por lo tanto reconoce la compilación del Software Arduino.

- La batería está diseñada para mantener al RTC funcionando por 10 años.

Las conexiones se hicieron de la siguiente manera: Arduino SCL con el pin A5, SDA con pin A4, VCC con el puerto $5 \mathrm{~V}$ y GND con el GND del Arduino.

\subsection{Módulo relé}

Para controlar componentes de alto voltaje 0 amperaje, como las bombas de agua, los cuales no pueden ser manejados directamente con Arduino, se utilizan relays o relés [4]. Estos dispositivos permiten controlar cargas de alto voltaje con una señal pequeña. El módulo es fabricado por Ningbo Songle Relay CoLtd, China, los cuales son capaces de manejar cargas de hasta $250 \mathrm{~V} / 10 \mathrm{~A}$. Cada canal posee aislamiento eléctrico por medio de un opto acoplador y un led indicador de estado. Funciona como un interruptor que por medio de una bobina y un electroimán, este acciona un juego de uno o varios contactos que permiten abrir o cerrar los circuitos eléctricos de la bomba de agua [2].

\section{Tabla 1. Especificaciones RTC-LCD}

\begin{tabular}{|c|c|c|}
\hline & Tierra (OV) & GND \\
\hline 2 & $\begin{array}{l}\text { Voltaje de Operación; } 5 \mathrm{~V}(4,7 \mathrm{~V} \\
-5,3 \mathrm{~V})\end{array}$ & VCC \\
\hline 3 & $\begin{array}{l}\text { Ajuste de contrastes: mediante } \\
\text { un potenciómetro (puede ser de } 1 \\
\mathrm{k} \Omega 10 \mathrm{k} \Omega \text { ) }\end{array}$ & VDD \\
\hline 4 & $\begin{array}{l}\text { Selección del registro, para } 0 \text { es } \\
\text { comandos y en } 1 \text { es para datos }\end{array}$ & Registro \\
\hline 5 & $\begin{array}{l}\text { Estado bajo para escribir y estado } \\
\text { alto para leer el registro }\end{array}$ & $\begin{array}{r}\text { Leer } \\
\text { escribir }\end{array}$ \\
\hline 6 & $\begin{array}{l}\text { Envía datos a los pinos de datos } \\
\text { cuando recibe un flanco de } \\
\text { bajada }\end{array}$ & Habilitar \\
\hline 15 & Backlight VCC (5 V) & Led+ \\
\hline 16 & Backlight Tierra $(0 \mathrm{~V})$ & Led- \\
\hline
\end{tabular}

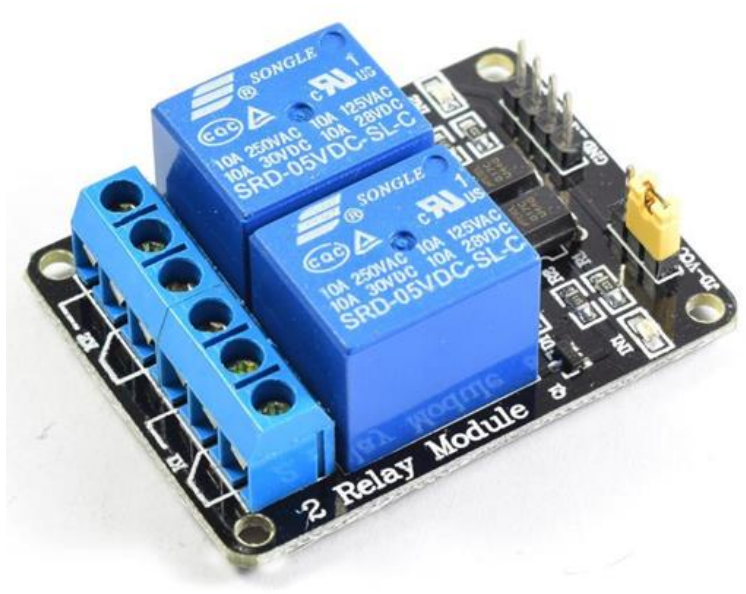

Figura 3: Imagen del módulo relé (tienda electrotecs Perú)

Conexión: 
Voltaje de operación: $5 \mathrm{~V}, \mathrm{VCC}$ con el puerto $5 \mathrm{~V}$ de la placa Arduino, GND con el GND de la placa Arduino y IN con el pin 13 de la placa Arduino.

\subsection{Pantalla LCD}

Los sistemas digitales embebidos como Arduino, PC u otros trabajan únicamente con lógica binaria (0 y 1), es por eso que para leer los datos empleamos pantallas o displays alfanuméricos LCD (Tabla 1). Esta pantalla nos sirvió para hacer correcciones en los distintos proyectos, sobre todo para el manejo de los módulos y procesamiento de datos como el tiempo estimado para el cultivo hidropónico.

EI LCD 16x2 posee 2 filas y 16 columnas de dígitos alfanuméricos como indica la tabla 1 , funciona con el controlador interno HD44780, que es un integrado muy utilizado y para el cual existe amplia documentación. Para conectar la pantalla LCD a nuestro Arduino/PIC se necesitaron 6 pinos: 2 de control y 4 de datos. En cuanto a la programación en Arduino se incluye por defecto la librería "LiquidCrystal", que incluye ejemplos de prueba.

Si bien es posible conectar directamente la pantalla LCD a nuestro Arduino, fue una buena opción utilizar un Adaptador LCD paralelo a serial I2C y de esa forma ahorrar pinos, trabajando con solo 2 pinos del puerto I2C [4].

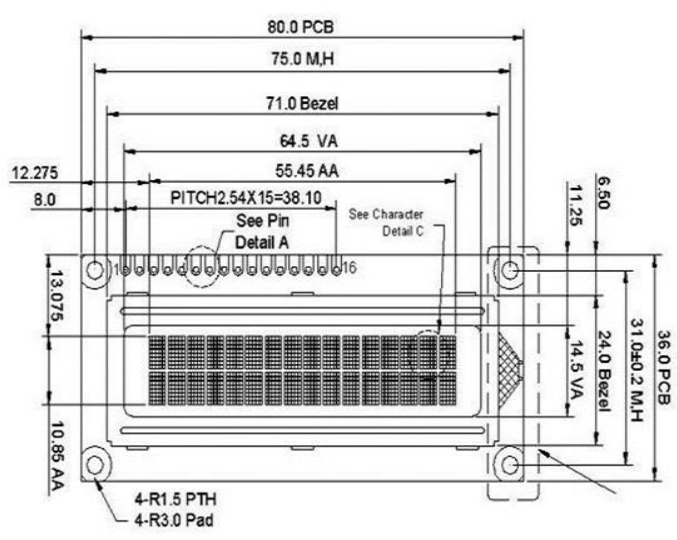

Figura 4. Pinos y medidas de la pantalla LCD [tienda Naylamp Mecatronics Perú]

Tabla 1. Función de los pinos de la pantalla LCD Conexión y funcionamiento:

\subsection{Materiales reciclables}

Para este proyecto ha sido posible utilizar materiales reciclables caseros como, molde de plástico reciclable de $16 \times 11 \mathrm{~cm}$, barras de silicona, cables de cobre para conexiones, estaño, etc.

\subsection{Bomba de agua}

La bomba de agua lleva la solución hasta los canales de cultivo, donde pasa el caudal permanentemente y con una pequeña cantidad de solución nutritiva la cual fluye hacia las raíces de las lechugas manteniéndolas nutridas, hidratadas y oxigenadas de esta manera en constante desarrollo [1].

\section{Resultados}

El sistema se instaló en la empresa "Hidroponía Ayacucho S.A." en la Comunidad de Mollepata Ayacucho, con 3842 de área y dimensiones $2 \mathrm{~m} \times 16$ $\mathrm{m}$ para cada parcela

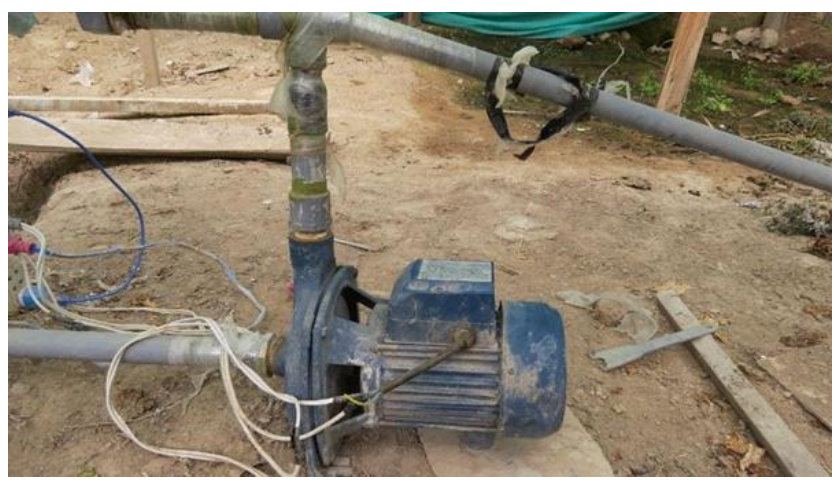

Figura 5. Imagen de la bomba de agua.

La bomba de agua tiene una capacidad de caudal máxima de $100 \mathrm{l} / \mathrm{min}$ con una altura máxima $36 \mathrm{~m}$, trabajando con una alimentación de $5,2 \mathrm{~A}$ y $220 \mathrm{~V}$.

\subsection{Conexión de sistema completo}

Se realiza las conexiones correspondientes de todos los componentes del sistema para el inicio de la prueba, según el diagrama de la figura 6 .

Estuvo en funcionamiento durante 3 meses, automatizando las dos bombas de agua instaladas en la base de los cultivos extrayendo el agua de un tanque de polietileno de 1100 litros para luego bombear a los cultivos de lechuga instalados a un metro de la base del suelo. 


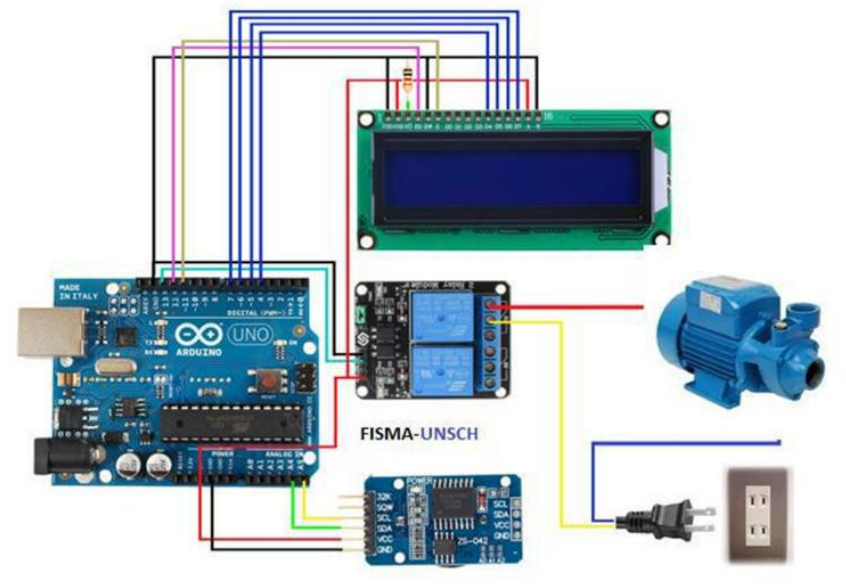

Figura 6: Imagen de la fase terminal del sistema de control automatizado y muestra las conexiones.
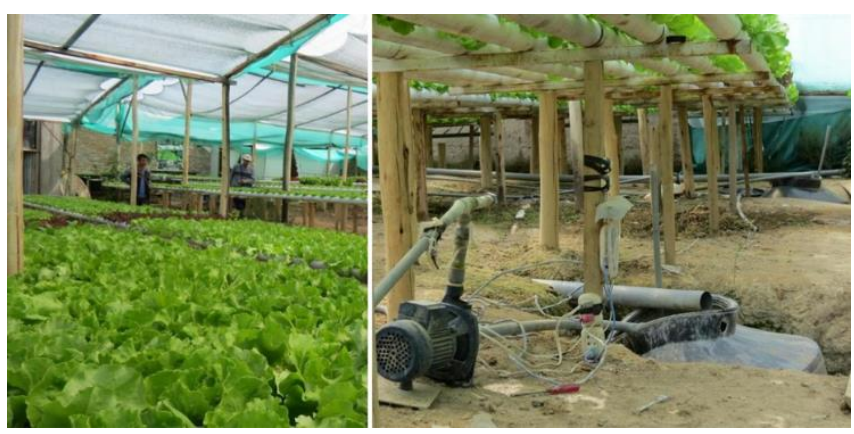

Figura 7: Imagen del área de cultivo hidropónico y los tanques de agua al ras del suelo.

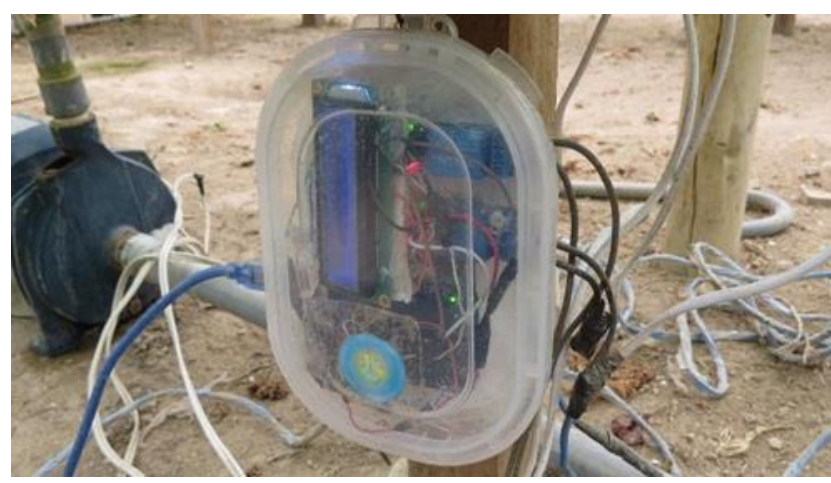

Figura 8: La Imagen muestra la instalación del sistema apoyado sobre caballetes de madera del cultivo hidropónico.

Este programa físicamente actúa a partir de la 1:00 de la mañana por 8 minutos luego activa nuevamente a las 6:00 am por 8 minutos y así sucesivamente a cada hora hasta las 10:00 pm, este proceso es cíclico durante el día y noche.

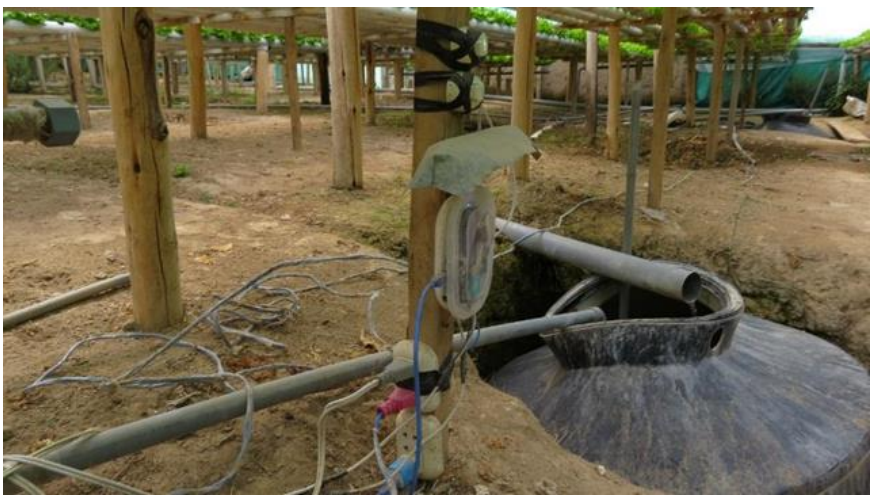

Figura 9: La Imagen muestra el sistema de control automatizado puesta en funcionamiento en el área de cultivo hidropónico.

De la información recopilada, el microclima interior del área de cultivo muestra que la temperatura del suelo y la del aire durante la noche alcanza valores de $10^{\circ} \mathrm{C}$, superiores de 4-5ㅇ $\mathrm{C}$ a la temperatura exterior, estos parámetros complementarios fueron considerados en el "diseño y construcción del sistema automatizado de control de bombas de agua". Para mantener seguro y aislado de la humedad.

La ventaja de nuestro proyecto con respecto a los sistemas industriales, es que podemos incorporar más relés para diferentes bombas de agua en el caso de ampliar el área de cultivo y graduar los tiempos de bombeo.

Para un óptimo funcionamiento de nuestro sistema se debe proveer de constante energía eléctrica, de otra forma se originaria una desactualización del tiempo programado, este problema se debe al tipo de precisión de los módulos de reloj insertados en el sistema automatizado.

\section{Conclusiones}

Se ha diseñado utilizando el micro controlador Arduino ATmega328P un sistema de control automatizado de bombeo de agua para un invernadero hidropónico e instalado en una cubierta de plástico a fin de protegerlo de la humedad como se muestra en la figura 8.

El sistema controlador Automatizado ya viene operando en un invernadero hidropónico, Mollepata-Ayacucho de una manera eficientemente desde hace 3 meses, logrando mejorar la producción del cultivo. 
Para el caso de futuros trabajos relacionados con este proyecto es necesario tener un abastecimiento permanente del fluido de agua y energía eléctrica, para garantizar la precisión de operación con el tiempo programado.

\section{Agradecimientos}

Al Centro de Energía Renovables de la Universidad Nacional de San Cristóbal de Huamanga (CERUNSCH), por su apoyo académico en la realización de este proyecto.

Al Comité Regional de Educación para el Desarrollo Internacional de Lanaudière (CREDIL-JOLIETTE) Canadá por su auspicio en la realización de este proyecto.

A la empresa Hidroponía - Ayacucho S.A. por permitirnos utilizar sus instalaciones para la implementación de nuestro proyecto.

A Fundación de Amparo à Pesquisa do Estado do Rio de Janeiro - FAPERJ.

\section{Anexo}

Para el funcionamiento del sistema se programó en el software Arduino, en lenguaje Java. El programa es el siguiente:

\#include <Wire.h> \#include "RTClib.h"

\#include < LiquidCrystal.h>

liquidCrystal Icd $(12,11,4,5,6,7)$;

RTC_DS3231 RTC;

int hora $=0$;

int minutos $=0$;

int segundos $=0$;

int salida=13;

int salida2 $=2$;

void setup () \{

Icd.begin $(16,2)$;

Inicializamos el puerto serie, wire y el módulo RTC

Serial.begin (9600);

Wire.begin ();

RTC.begin ();

Si quitamos el comentario de la línea siguiente, se ajusta la hora y la fecha con la del ordenador

RTC.adjust (Date Time (_DATE_, _ TIME__));

PinMode (salida, OUTPUT);

\}

void loop () \{

DateTime now = RTC.now ();

hora $=$ (now.hour (),DEC);

minutos $=$ (now. minute ()$, \mathrm{DEC})$;

segundos=(now.second(),DEC);

Imprimimos el dia

Icd.setCursor $(0,0)$;
Icd.print(now.day(), DEC);

Icd.print('/');

Imprimimos el mes

Icd.print(now.month(), DEC);

Icd.print('/');

Imprimimos el año

Icd.print(now.year(), DEC);

Icd.print(' ');

Imprimimos la hora

Icd.setCursor $(0,1)$;

Icd.print(now.hour(), DEC);

Icd.print(':');

Imprimimos los minutos

Icd.print(now.minute(), DEC);

Icd.print(':');

Imprimimos los segundos

Icd.print (now.second(), DEC);

Icd.setCursor $(11,0)$;

Icd.print("FOCO");

if $($ now.hour ()$==1$ \&\& now.minute ()$==00 \& \&$

now.second ()$==00$ )

\{digitalWrite(salida,LOW);

Icd.setCursor(12,1);

lcd.print(" ON ");

delay (480000);

else if (now.hour ()$==6 \& \&$ now.minute ()$==00 \& \&$ now. second ()$==00$ )

\{ digitalWrite(salida,LOW); Icd.setCursor(12,1); Icd.print(" ON "); delay(480000); \}

else if (now.hour ()$==7 \& \&$ now. minute ()$==00 \& \&$

now. second ()$==00$ )

\{digitalWrite(salida,LOW);

Icd.setCursor(12,1);

Icd.print(" ON ");

delay (480000); $\}$

else if (now.hour ()$==8$ \&\& now.minute ()$==00$ \&\&

now. $\operatorname{second}()==00)$

\{ digitalWrite(salida,LOW); Icd.setCursor(12,1);

lcd.print(" ON ");

delay (480000); \}

else if (now.hour ()$==9 \& \&$ now.minute ()$==00 \& \&$ now. second ()$==00$ )

\{ digitalWrite(13,LOW); Icd.setCursor(12,1);

lcd.print(" ON ");

delay $(480000) ;\}$

else if (now.hour ()$==10 \& \&$ now.minute ()$==00 \& \&$

now. second ()$==00$ )

\{digitalWrite(salida,LOW);

Icd.setCursor(12,1);

Icd.print(" ON ");

delay $(480000) ;\}$

else if (now.hour ()$==10 \& \&$ now.minute ()$==30$ \&\&

now. second ()$==00$ )

\{digitalWrite(salida,LOW);

Icd.setCursor(12,1);

lcd.print(" ON "); 
$\operatorname{delay}(480000) ;\}$

else if (now.hour ()$==11 \& \&$ now.minute ()$==00 \& \&$

now.second ()$==00$ )

\{digitalWrite(salida,LOW);

Icd.setCursor(12,1);

lcd.print(" ON ");

delay (480000); \}

else if $($ now.hour ()$==11 \& \&$ now. minute ()$==30 \& \&$

now. second ()$==00$ )

\{ digitalWrite(salida,LOW); Icd.setCursor(12,1); lcd.print(" ON ");

delay (480000); \}

else if (now.hour ()$==12 \& \&$ now. minute ()$==00 \& \&$

now.second ()$==00$ )

\{digitalWrite(salida,LOW);

Icd.setCursor(12,1);

lcd.print(" ON ");

delay $(480000) ;\}$

else if (now.hour ()$==12 \& \&$ now.minute ()$==30 \& \&$

now. $\operatorname{second}()==00$ )

\{digitalWrite(salida,LOW);

Icd.setCursor(12,1);

Icd.print(" ON ");

delay (480000); $\}$

else if (now.hour ()$==13 \& \&$ now.minute ()$==00 \& \&$

now. second ()$==00$ )

\{digitalWrite(salida,LOW);

Icd.setCursor(12,1);

Icd.print(" ON ");

delay (480000); \}

else if (now.hour ()$==13 \& \&$ now.minute ()$==30$ \&\&

now.second ()$==00$ )

\{ digitalWrite(salida,LOW); Icd.setCursor(12,1); lcd.print(" ON ");

delay $(480000) ;\}$

else if (now.hour ()$==14 \& \&$ now.minute ()$==00 \& \&$

now.second ()$==00$ )

\{digitalWrite(salida,LOW);

Icd.setCursor(12,1);

Icd.print(" ON ");

delay $(480000) ;\}$

else if (now.hour ()$==14$ \&\& now. minute ()$==30$ \&\&

now.second ()$==00$ )

\{ digitalWrite(salida,LOW); Icd.setCursor(12,1);

Icd.print(" ON ");

delay (480000); \}

else if (now.hour ()$==15 \& \&$ now.minute ()$==00 \& \&$

now. $\operatorname{second}()==00$ )

\{ digitalWrite(salida,LOW); Icd.setCursor(12,1); Icd.print(" ON "); delay(480000); \}

else if (now.hour ()$==15 \& \&$ now.minute ()$==30 \& \&$ now.second ()$==00$ )

\{ digitalWrite(salida,LOW); Icd.setCursor(12,1);

Icd.print(" ON "); delay(480000); \} else if (now.hour ()$==16 \& \&$ now.minute ()$==00 \& \&$ now. $\operatorname{second}()==00$ )

\{ digitalWrite(salida,LOW); Icd.setCursor(12,1); Icd.print(" ON "); delay(480000);

else if (now.hour ()$==16 \& \&$ now.minute ()$==00 \& \&$ now. second ()$==00$ )

\{digitalW rite(salida,LOW);

Icd.setCursor(12,1);

Icd.print(" ON ");

delay (480000); \}

else if (now.hour ()$==16 \& \&$ now. minute ()$==30$ \&\& now. second ()$==00$ )

\{ digitalWrite(salida,LOW); Icd.setCursor(12,1);

lcd.print(" ON ");

delay (480000); \}

else if (now.hour ()$==18 \& \&$ now.minute ()$==00 \& \&$ now. second ()$==00$ )

\{ digitalWrite(salida,LOW); Icd.setCursor(12,1); lcd.print(" ON ");

delay $(480000) ;\}$

else if (now.hour ()$==22 \& \&$ now. minute ()$==00$

$\& \&$ now.second ()$==00$ )

\{ digitalWrite(salida,LOW); Icd.setCursor(12,1); Icd.print(" ON "); delay(480000); \}

else

\{digitalWrite(salida,HIGH);

Icd.setCursor(11,1); Icd.print("OFF"); \}\}

\section{Referencias}

[1] Gilsanz, Juan C. Hidroponia. No. CIDABSB321-G5h. Instituto Nacional de Investigación Agropecuaria (Uruguay)., 2007.

[2] Vergara, Joseph, Robinson Molina, and César Machado. "Prototipo para control automático de nivel y caudal de líquidos." Ingenius. Revista de Ciencia y Tecnología 6 (2011): 918.

[3] HERRADOR, Rafael Enríquez. Guía de usuario de Arduino. Universidad de Córdoba, 2009, vol. 13.

Naylamp Mechatronics, S. A. C. "Naylamp

[4] Mechatronics."línea].Available:https://naylam pmechatronics.com/inalambrico/115-modulogsm-sim8001. html (2018).

MUNDIAL, Banco. Agricultura para el

[5] desarrollo. Informe sobre el desarrollo mundial, v. 2008, p. 19, 2008.).

Kushner, David. "The making of arduino."

[6] IEEE spectrum 26 (2011). 